\title{
Integrative testis transcriptome analysis reveals differentially expressed miRNAs and their mRNA targets during early puberty in Atlantic salmon
}

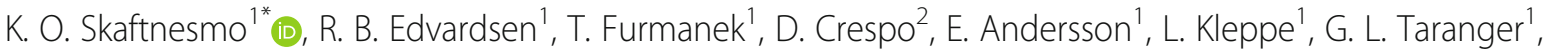
J. Bogerd ${ }^{2}$, R. W. Schulz ${ }^{1,2}$ and A. Wargelius ${ }^{1}$

\begin{abstract}
Background: Our understanding of the molecular mechanisms implementing pubertal maturation of the testis in vertebrates is incomplete. This topic is relevant in Atlantic salmon aquaculture, since precocious male puberty negatively impacts animal welfare and growth. We hypothesize that certain miRNAs modulate mRNAs relevant for the initiation of puberty. To explore which miRNAs regulate mRNAs during initiation of puberty in salmon, we performed an integrated transcriptome analysis (miRNA and mRNA-seq) of salmon testis at three stages of development: an immature, long-term quiescent stage, a prepubertal stage just before, and a pubertal stage just after the onset of single cell proliferation activity in the testis.

Results: Differentially expressed miRNAs clustered into 5 distinct expression profiles related to the immature, prepubertal and pubertal salmon testis. Potential mRNA targets of these miRNAs were predicted with miRmap and filtered for mRNAs displaying negatively correlated expression patterns. In summary, this analysis revealed miRNAs previously known to be regulated in immature vertebrate testis (miR-101, miR-137, miR-92b, miR-18a, miR-20a), but also miRNAs first reported here as regulated in the testis (miR-new289, miR-30c, miR-724, miR-26b, miR-new271, miR217, miR-216a, miR-135a, miR-new194 and the novel predicted n268). By KEGG enrichment analysis, progesterone signaling and cell cycle pathway genes were found regulated by these differentially expressed miRNAs. During the transition into puberty we found differential expression of miRNAs previously associated (let7a/b/c), or newly associated (miR-15c, miR-2184, miR-145 and the novel predicted $n 7 a$ and $b$ ) with this stage. KEGG enrichment analysis revealed that mRNAs of the Wnt, Hedgehog and Apelin signaling pathways were potential regulated targets during the transition into puberty. Likewise, several regulated miRNAs in the pubertal stage had earlier been associated (miR-20a, miR-25, miR-181a, miR-202, let7c/d/a, miR-125b, miR-222a/b, miR-190a) or have now been found connected (miR-2188, miR-144, miR-731, miR-8157 and the novel n2) to the initiation of puberty.
\end{abstract}

Conclusions: This study has - for the first time - linked testis maturation to specific miRNAs and their inversely correlated expressed targets in Atlantic salmon. The study indicates a broad functional conservation of already known miRNAs and associated pathways involved in the transition into puberty in vertebrates. The analysis also reveals miRNAs not previously associated with testis tissue or its maturation, which calls for further functional studies in the testis.

Keywords: miRNA, Aquaculture, Salmo salar, Integrative analysis

* Correspondence: kai.ove.skaftnesmo@imr.no

${ }^{1}$ Institute of Marine Research, Postboks 1870 Nordnes, 5817 Bergen, Norway

Full list of author information is available at the end of the article

(c) The Author(s). 2017 Open Access This article is distributed under the terms of the Creative Commons Attribution 4.0 International License (http://creativecommons.org/licenses/by/4.0/, which permits unrestricted use, distribution, and reproduction in any medium, provided you give appropriate credit to the original author(s) and the source, provide a link to the Creative Commons license, and indicate if changes were made. The Creative Commons Public Domain Dedication waiver (http://creativecommons.org/publicdomain/zero/1.0/) applies to the data made available in this article, unless otherwise stated. 


\section{Background}

In vertebrates, the brain-pituitary system has evolved as the master regulator of pubertal development and adult functioning of the gonads. Fish share many homologies with other vertebrates, also regarding the basic building blocks of the brain-pituitary-gonad (BPG) axis [1-3]. In salmon farming, precocious puberty generates animal welfare problems and inflicts economic damage, since puberty is associated with an increased susceptibility to disease, affects flesh quality, decreases growth and causes hypo-osmoregulatory problems. Precocious puberty is a male-specific problem, since the high energetic requirement of female puberty usually does not allow it to occur until females are harvested [4]. This applied background complements the general scientific interest in understanding the molecular mechanisms implementing testis maturation in animals.

Several proteins in fish testis regulate the initial steps of spermatogenesis such as Amh, Gsdf, Igf3 and Insl3 in eel [5, 6], rainbow trout [7] and zebrafish [8-10]. A few transcriptome studies in fish have reported changes in testicular mRNA levels and provided information on the potential involvement of genes and pathways regulating pubertal testis maturation [11-14]. In addition to mRNAs, miRNAs impact every regulatory pathway in animals [15], while there is limited information on the potential roles of miRNAs in testis maturation in fish [16]. miRNAs are short ( 19-23 nucleotides) palindromic noncoding RNA species that specifically target and bind 3'-UTR regions of mRNAs, resulting in posttranscriptional silencing via accelerated mRNA decay and translational repression in a mechanism mediated by Argonaute family members [17]. Hence, miRNAs regulate the abundance and usage of expressed mRNAs. Also a given miRNA can target more than one mRNA, since different mRNAs can share the region complementary to the miRNA's seed region [18].

The significance of miRNAs for testicular function has been demonstrated in several studies and work in mice has identified miRNA processing enzymes in testis as essential [16, 19-22]. Additional studies have also established functions for specific miRNAs in regulating spermatogenesis, including the miR-17-92 family [23], the testis-enriched and sexually-dimorphic miR-140 [24], the retinoic acid (RA)-induced $m i R-146$, and the clustered $m i R-221$ and $m i R-222$ [25-27]. Several studies described miRNAs in commercial fish species such as yellow catfish [28], halibut [29, 30] and Atlantic salmon [31-33]. The latter studies described the miRNA repertoire in Atlantic salmon tissues, but did not investigate miRNA expression in testis. The first study dealing with the testicular miRNA repertoire in salmonids examined immature and fully mature testis of rainbow trout [34]. However, comparing the start and end points of a developmental process stretching over several months provides a limited amount of specific information on the potential functional context of changes in miRNA expression.

In view of our interest in early stages of spermatogenesis and testis maturation, we concentrate on miRNAs of potential relevance for initiating puberty. To identify suitable testis tissue samples, we monitored a group of 2-year-old salmon for 10 months and defined three groups based on morphological, physiological, and molecular parameters: immature males, males a few weeks before (prepubertal), and males shortly after the initiation of testis maturation (pubertal). Since the main known function of miRNAs is to regulate the use of expressed mRNAs, we decided to analyze, by RNA sequencing, both mRNA and miRNA repertoires in the same set of samples. The potential interaction of miRNAs with target mRNAs was then investigated, by examining the expression correlation of predicted targets of differentially expressed miRNAs. With this integrated approach mRNA targets predicted by the miRmap algorithm [35] were refined to targets showing an inversely correlated expression to their respective targeting miRNA. Utilizing this approach, we have identified sets of testicular miRNAs targeting pathways known to be involved in preventing or promoting differentiation processes in the vertebrate testis in addition to in this respect potential new miRNA targets.

\section{Results}

\section{Sample characterization}

To identify cues controlling molecular pathways associated with puberty in Atlantic salmon, we characterized differentially expressed miRNAs and mRNAs in samples from immature, prepubertal and pubertal testis. To select suitable testis tissue samples, we investigated several maturity associated parameters, including the gonadosomatic index (GSI), plasma levels of 11ketotestosterone $(11-\mathrm{KT})$ and the relative expression levels of $a m h$ and igf3 in testis (Fig. 1A). These measurements were complimented by histological analysis of germ cell development and immunohistochemical analysis of testicular proliferation activity. These combined analyses allowed selecting males for assignment of their testis tissue samples into three groups; an immature stage, a prepubertal stage showing limited proliferation activity and a pubertal stage showing elevated single cell proliferation activity of both, spermatogonia and Sertoli cells (Fig. 1B). Based on this grouping, we decided to in depth characterize differentially expressed miRNAs and their inversely correlated targets in Atlantic salmon testis (Fig. 1C). A more detailed description of the tissue sample donors can be found in Additional file 1. 


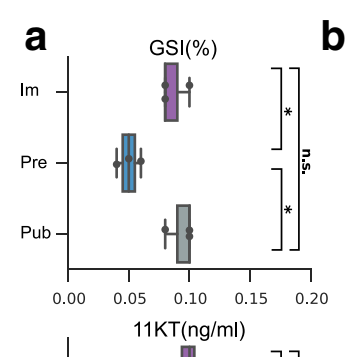

\section{b}
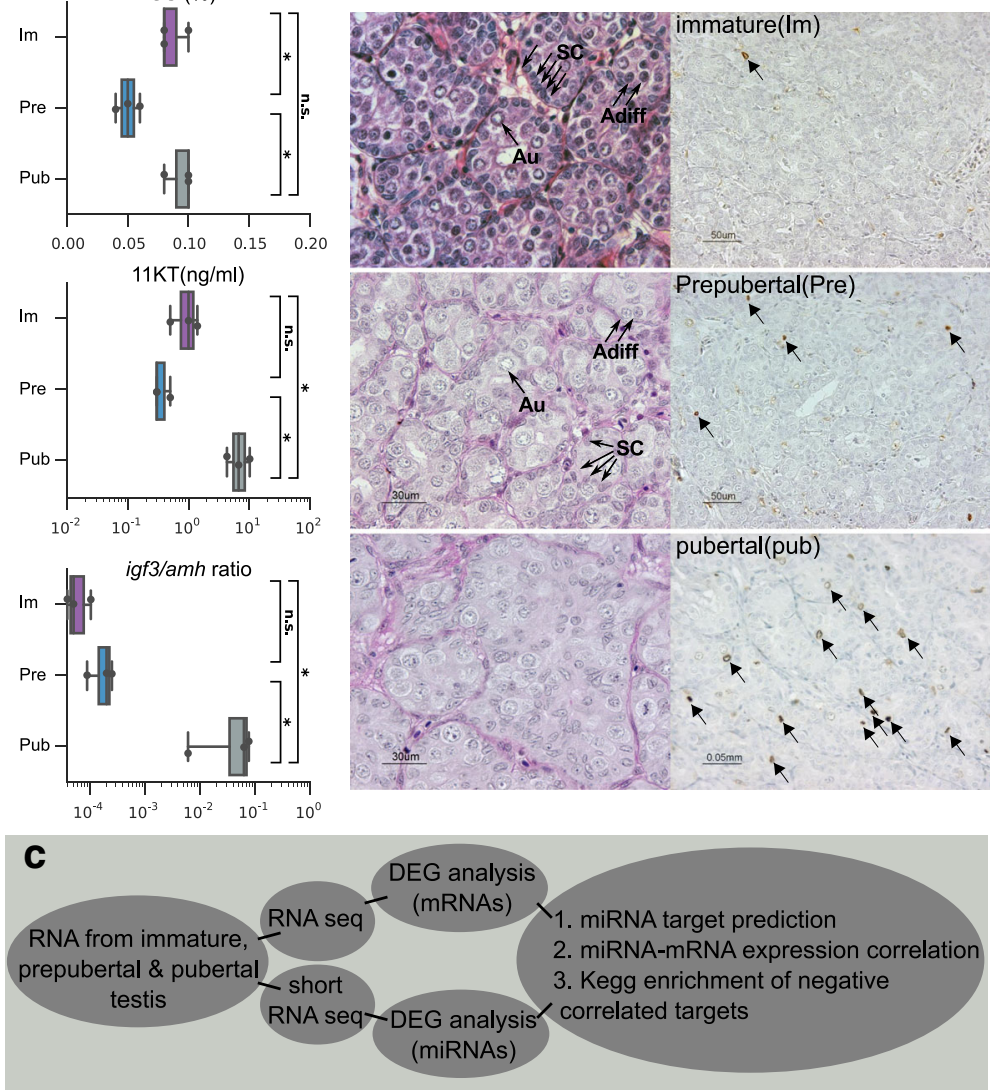

Fig. 1 Description of samples and analysis procedure. (a) Gonadosomatic index (GSI, \%; upper), 11-ketotestosterone levels (11 KT, ng/ml; center) and the ratio of gene expression for igf3/amh (lower) in testis samples used for miRNA and mRNA-seq analysis. Statistical significant differences are marked with asterisk (*: $p<=0.05$, n.s.: non significant, according to Wilcoxon rank-sum statistic). (b) Representative histology (hematoxylineosin combined with periodic acid according to Schiff; HE/PAS; left column) and proliferation activity (immunocytochemical detection of phosphorylated histone $\mathrm{H3}$; $\mathrm{pH}$ 3; right column, marked with triangles) in immature (Im), prepubertal (Pre) and pubertal (Pub) testis samples. Abbreviations: Au = type A undifferentiated spermatogonia, and Adiff = type A differentiating spermatogonia, SC = Sertoli Cells (c) Illustration of experimental setup and analysis pipeline used in this paper

\section{Integrated expression analysis}

Differential miRNA expression analysis revealed five expression clusters (I-V, Fig. 2). We analyzed correlations between expression profiles from the miRNA expression dataset with expression profiles of predicted and differentially expressed targets from an mRNA expression dataset derived from the same samples. Differentially expressed mRNAs are provided in Additional file 2. In a similar approach as undertaken by others [36-38], we focused on analyzing inverse correlated miRNA -mRNA target pairs with the assumption that high confident miRNA-mRNA targeting pairs display a negative correlation as the result of mRNA deadenylation and destabilization which alongside with translational repression is considered the mechanism of miRNA repression [39]. This analysis revealed that the $20 \%$ best scoring predicted targets for each miRNA (Additional file 2), when refined by considering only negatively correlated target mRNAs (Pearson correlation $<=-0.5$ ), had specific enrichment of KEGG pathways (Fig. 3) associated to each miRNA expression cluster (Fig. 2, Additional file 2).

Cluster I contained six miRNAs (miR-30c, miR-new289, miR-137, miR-724, miR-101a and miR-26b) that had a higher expression in the immature and prepubertal stages than in the pubertal stage. By KEGG enrichment, cluster I displayed potential target mRNAs representing genes involved in cell cycle and metabolic pathways (Figs 3,4). Of the genes associated to cell cycle, several genes were related to mitosis ( $c d c 27$, $c d c 23)$, both meiosis and mitosis $(f z r 1, b u b 1 b)$ or meiosis (cdkn1a) [40-42]. Interestingly, Cdkn1a has previously also been associated with Fsh-induced stimulation of spermatogonial differentiation in zebrafish [12]. Other genes possibly related to the maturing gonad are wee1- $b$ and $e 2 f 2$ which encode proteins associated with stem cell renewal, cell proliferation and controlled proliferation 

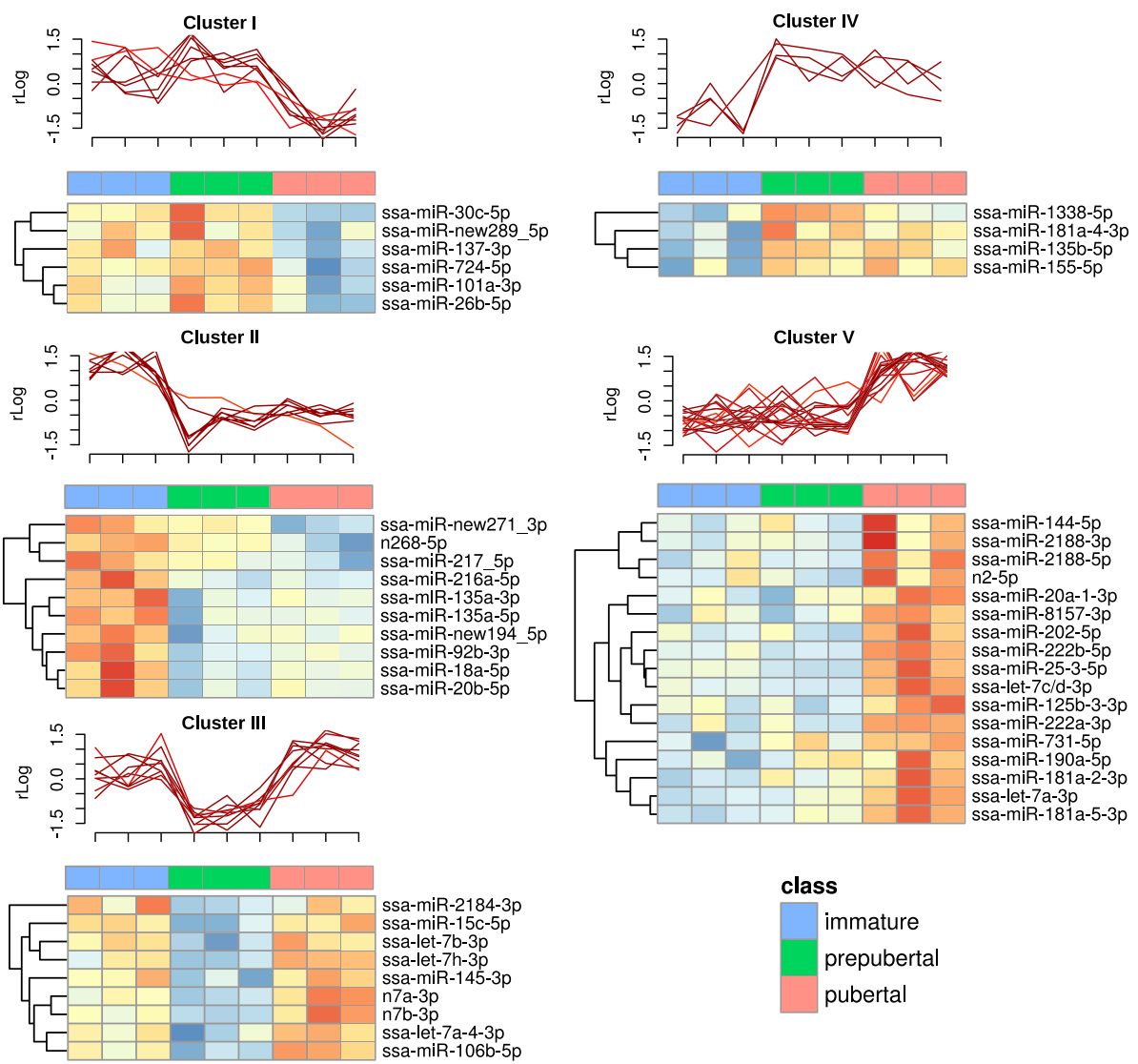

Fig. 2 Hierarchical clusters of miRNAs that are differentially expressed in salmon testis. Heatmap displaying 5 hierarchical clusters of miRNAs that are differentially expressed in salmon testis during onset of puberty (values are depicted as DESeq2 normalized rlog expression values (log2 scale). On the $x$-axis, the type of sample is illustrated with color codes (immature-blue, prepubertal-green, pubertal-pink). Above the heatmaps, expression profiles are depicted. The k-means profile plots highlighted in light-red correspond to less similar profiles. On the y-axis DESeq2 normalized rlog values are plotted (log2 scale)

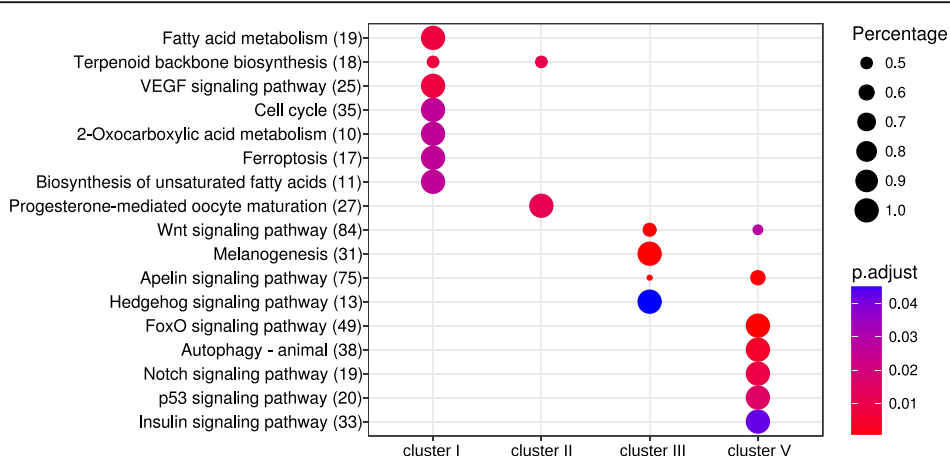

Fig. 3 KEGG pathway enrichment of target mRNAs associated with miRNA clusters. Functional enrichment of the 20\% top scoring miRmap target predictions for each miRNA where an association were supported by a negatively correlated mRNA expression profile (Pearson correlation $<=-0,5)$. Dots represent KEGG term enrichment where the color coding indicates the magnitude of the adjusted p-values (p.adj). Adjusted p-values ranges from 0.0415581719069 to $5.5547769425 \mathrm{e}-08$, where a red color indicates a lower $p$-value and a blue color represents a higher p-value). The sizes of the dots represent the percentage of each row (KEGG category). On the $y$-axis in parentheses after the KEGG term is the number of genes found in the pathway. Cluster IV is not represented as target genes for this cluster of miRNAs produced no KEGG term enrichment 


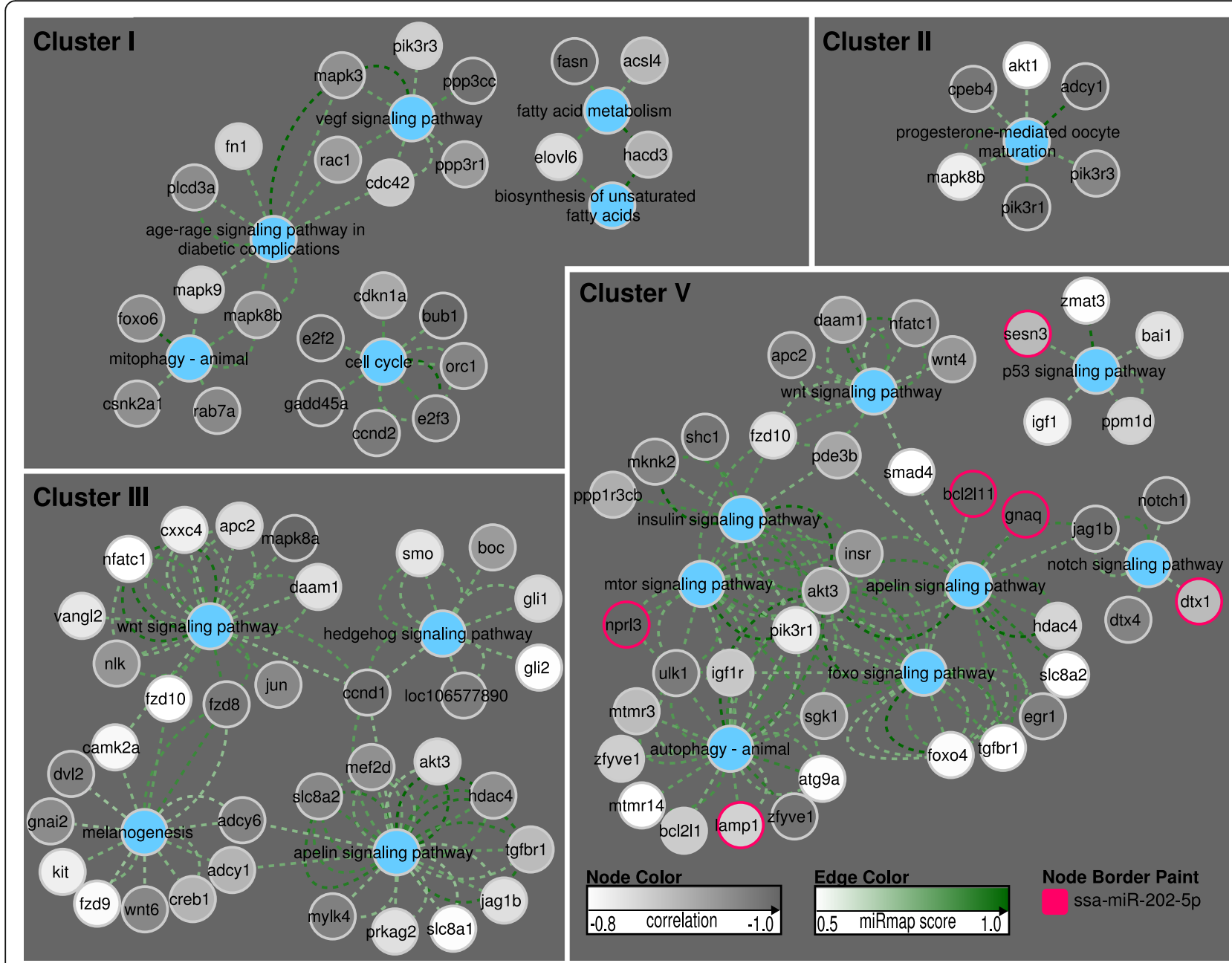

Fig. 4 Target genes in KEGG pathways. Single genes/pathways significantly associated with miRNA clusters. A Cytoscape network rendering of enriched KEGG pathways and their miRNA targeted gene members. The color of the nodes is assigned according to the strength of the negative Pearson correlation of expression profiles of its targeting miRNA (Pearson correlation -1 to -0.8 , dark grey to white). The dotted lines connecting the nodes (edges) are colored from light green to green according to the corresponding strength of the miRNA target prediction (miRmap target prediction score, white to green). Red rings around nodes in cluster $V$ are depicting genes predicted to be regulated by miR-202. Raw data can be manually inspected in Additional file 2 (correlation and miRmap scores). Cluster IV is not represented as target genes for this cluster of miRNAs produced no KEGG term enrichment

through apoptosis in other animals [43, 44]. High scoring mRNA target predictions of the cell cycle pathway were all predicted as targets of either miR-724, miR-137, $m i R-30$ or miR-101a. Two of these miRNAs have been associated with testis function previously, since miR-101 and $m i R-137$ were negatively regulated in response to RA-induced spermatogenesis in dogs [27].

Cluster II contained miRNAs with a higher expression in the immature relative to the prepubertal and pubertal stages. This cluster contained ten miRNAs (miR-new271, miR-217, miR216a, miR-135a, miR-new-194, miR$92 b, m i R-18 a, m i R-20 b$ and the novel predicted miRNA n268). KEGG enrichment analysis revealed possible target genes belonging to the progesterone-mediated oocyte maturation pathway (Figs 3 and 4). Some of the
miRNAs found in this cluster have previously been associated with a function in the testis, like miR-18a and $m i R-92 b$ which belongs to the miR-17-92 cluster that is essential for spermatogenesis in mice [23]. miR-92b is also highly abundant in zebrafish gonads and spermatozoa [45]. The other miRNAs found in cluster II, miRnew271, miR-217, miR-216a and miR-135a have, however, not been linked yet to a function in the testis.

Cluster III included nine miRNAs (miR-2184, miR-15c, let-7b/h/a, miR-145, miR-106b, and the novel predicted $n 7 a$ and $b$ ) that displayed a drop in expression during the prepubertal stage. Their reduced expression during this transitional stage suggests that suppression of targeted mRNAs is alleviated at this stage, potentially allowing entry into maturation. The KEGG enrichment 
of miRNA targets for cluster III involved Wnt, Hedgehog, Apelin and Melanogenic signaling (Figs 3 and 4). High scoring targets in these pathways displayed target relations to the following miRNAs; the novel predicted miRNAs $n 7 a$ and $n 7 b$, let $7 a / b / h$, miR-106b and $m i R-$ 2184. let-7a and let-7b have also been demonstrated to peak in expression in rat testis during puberty, implicating a conserved modulating role for these miRNAs in vertebrate puberty [46]. $m i R-106 b$ has been shown previously to be downregulated during RA-induced spermatogonial differentiation in mouse [23]. miR-2184 has been found previously in medaka testis, but has not been linked to puberty so far [47].

Cluster IV contained only 4 miRNAs $(m i R-1338$, miR$181 a, m i R-135 b$ and $m i R-155)$ that displayed a lower expression in immature but higher expression in the prepubertal and pubertal testis. While no predicted enriched pathways were retrieved for this cluster, its expression profile is compatible with the notion that miRNAs of cluster IV may have a role in suppressing targets involved in keeping the testes in an immature state.

Cluster V consisted of a relatively large group of 17 miRNAs (miR-144, miR-2188, miR-20a, miR-8157, miR202, miR-222b, miR-25, let7c/d/a, miR-125b, miR-222a, miR-731, miR-190a, miR-181a and the novel $n 2$ ), characterized by a lower expression in immature and prepubertal testis. Among potentially targeted pathways regulated by miRNAs in this cluster, we found mRNAs associated with the interconnected Apelin, Wnt, FoxO, insulin, mTor, Notch and Autophagy signaling pathways (Fig. 4, listed in Additional file 2). Key miRNAs including the novel predicted miRNA $n 2$, let7 $a$ and $c$, miR-125b, miR144, miR-181, miR-190a, miR-202, miR-20a, miR-2188, $m i R-731$ and $m i R-8157$ were supported by both a high prediction score and a negative correlation to targets of these pathways (Fig. 4, Additional file 2). Interestingly, miR-125b, let7a and $c$ have been found expressed in dog testis [27]. $m i R-202$ is expressed in Sertoli cells and a knockout model in mice has demonstrated that this miRNA as essential for spermatogonial stem cell survival [48]. miR-202 is also highly abundant in the testis of trout, representing $20 \%$ of all microRNAs found in testis tissue [34], further underscoring the significance of this miRNA. We observed in our dataset that miR-202 is targeting many genes in the above-mentioned pathways including dtx1, gnaq, bcl2l11 and nprl3 (highlighted with red rings in Fig. 4) in addition to tgfbr-1 (Additional file 2). Tgfbr-1 and smad4 are key signaling transmitters of the Tgf- $\beta$ superfamily ligands such as the testis maturation inhibiting factor Amh $[1,49,50]$, and are in our data predicted as potential targets of $m i R-181 a$ and $m i R-190 a$, respectively (Additional file 2, cluster V); $m i R-190 a$ was also regulated during bovine oocyte maturation [51]. Jag1 and Notch1 are single pass transmembrane receptors in the
Notch signaling pathway that in our data are predicted targets of $m i R-144$ and let $7 c$, respectively (Fig. 4 and Additional file 2). Additionally, we observed that igf1 and igfr 1 , in mice identified as stimulating Sertoli cell proliferation [52], are potential targets of let7a and c, miR-2188, miR-202 and miR-125b (Additional file 2, cluster V). Two miRNAs in cluster $\mathrm{V}$, miR-25 and miR-181a, were abundant in zebrafish spermatozoa [45], while $m i R-2188$ is expressed in halibut gonads but has not been associated with testis maturation yet [30]. miR-20a, a sexually dimorphic miRNA, is a possible target of DMRT-1 in the testis of Nile tilapia [53]. In addition to miRNAs that previous work has linked to testis maturation, we found miRNAs in cluster $\mathrm{V}$ that have not been reported so far as linked to testis maturation, such as $m i R-731, m i R-8157$ and the novel predicted $n 2$.

\section{Discussion}

While a previous study has described differences in miRNA repertoires between immature and fully mature testis in a related species, the rainbow trout [34], we were interested in finding triggers of maturation through monitoring testicular RNA repertoires in a narrower time window around the initiation of testis maturation and contrasting this with immature testis months away from the potential start of puberty. For the selection of testis tissues, our criteria included the measurement of several parameters involved in determining maturity such as plasma $11 \mathrm{KT}$ /testosterone levels, gonadosomatic index (GSI) and testicular expression of igf3 and $a m h$ in conjunction with histological and immunohistochemical evaluation of proliferation. Recombinant zebrafish Igf3 stimulated [9] while recombinant zebrafish Amh inhibited spermatogonial differentiation [10] and androgen treatment of immature salmon promoted spermatogonial differentiation associated with elevated igf3 but reduced amh testicular transcript levels [54]. The ratio of these transcripts was therefore used as a measure of maturity. These combined analyses allowed selecting males for assignment of their testis tissue samples into three groups; an immature, quiescent stage months before the potential start of maturation; a prepubertal stage showing little proliferation activity; and a pubertal stage showing elevated single cell proliferation activity of both, spermatogonia and Sertoli cells (Fig. 1B), just after the start of seasonal testis growth. An integrative miRNAmRNA transcriptome analysis of these samples revealed specific miRNA expression patterns as well as KEGG pathway enrichment of negatively correlated and high confidence predicted mRNA targets of these miRNAs.

The miRNAs belonging to expression cluster I showed a higher expression in the immature and prepubertal stages. We suspect therefore that these miRNAs might mediate suppression of puberty-inducing mRNAs. By 
KEGG enrichment analysis, we found the predicted targets of miRNAs in this cluster to be enriched for mRNAs that code for proteins involved in the cell cycle pathway. This observation seems consistent with a down-regulation of these mRNAs during a stage were the gonad remains quiescent with a low cell division and metabolic activity.

miRNAs in cluster II are preferentially expressed in the immature stage and might thus also act to suppress maturation inducing mRNA targets. As these miRNAs displayed a sharp decrease in expression past the immature stage they are, however, more likely to target a subset of mRNAs that are released from miRNA-mediated inhibition earlier than those targeted by cluster I. The mRNAs enriched in the progesterone-mediated oocyte maturation pathway might be relevant in this respect, since signaling by the progestin $17 \alpha, 20 \beta$-dihydroxy-4pregnen-3-one (DHP) through this pathway is an important step in stimulating the spermatogonial phase in fish spermatogenesis $[55,56]$. In adult zebrafish, DHP treatment doubled the testis weight, increased spermatogonial proliferation/differentiation and affected expression of several growth factor genes [57]. Targeting of specific transcripts in the progesterone pathway by either miR-135a, miR-92, n268 or miR-20b as observed in our data (Fig. 4, Additional file 2) might thus act to prevent the gonad from entering puberty.

miRNAs in cluster III displayed a dip in expression in the prepubertal stage and we therefore speculate that these miRNAs target mRNAs that need to be transiently derepressed in order to allow entry into puberty. The mRNA targets of these miRNAs were associated with the Wnt, Hedgehog and the Apelin signaling pathways. Interestingly, the Apelin pathway is noted to contain also members of the Tgf- $\beta$ pathway, which in conjunction with both the Wnt and Hedgehog signaling pathways had been associated with proliferation activity in and growth of the zebrafish testis [12]. Studies on rat testis have additionally shown that Hedgehog signaling promotes the survival of germ cells and can be suppressed by FSH, a key endocrine regulator of germ cell differentiation [58]. By targeting gli1 ( $n 7 a)$, gli2 (let-7 h) and smo (let7b, miR-106b), all being downstream mediators of Hedgehog signaling, these miRNAs might modulate the Hedgehog pathway during entry into puberty (Additional file 2: Table S1).

Cluster IV and V contained miRNAs that had a higher expression towards the onset of maturation. These miRNAs we thus suspect to be involved in suppressing processes that would prevent the onset of testis maturation. We found no enriched pathways for targets of miRNAs expressed in cluster IV. Considering the relatively few miRNAs in this cluster it is possible that their cumulative targets were too few to result in any pathway enrichment. Cluster V, however, was enriched for target mRNAs associated with the interconnected Apelin, Wnt, FoxO, insulin, mTOR, Notch and Autophagy signaling pathways (Fig. 4, Additional file 2). Smad4 and tgfbr-1 which both are central to signaling by the Tgf $\beta$ superfamily ligands such as the testis maturation inhibiting hormone Amh [1, 49, 50], were predicted targets of $m i R-190 a$ and $m i R-181 a$ respectively. Tgfbr-1 was additionally found targeted by miR-202 (Additional file 2), a miRNA that recently has been found to be essential for spermatogonial stem cell survival [48]. The targets of miR-202 remain largely undefined. Besides tgfbr 1 , only bcl2l11, a pro-apoptotic protein, could be linked to a function in spermatogenesis [59]. For nprl3 and $d t x 1$, negative regulators of mTOR and Notch signaling, respectively, no function has been reported previously in the testis although both pathways have been implicated in regulation of maturation [12, 60]. While gnaq is expressed in the sheep testis [61], its function is unknown.

We observe also a possible involvement of miRNAs in modulating insulin signaling, by targeting igf1 and igfr 1 . It is possible thus that these miRNAs in salmon have an important role in modulating Igf action during puberty. Interestingly, an experimental support for a let-7/igf1 target regulation in gonads has been determined in mice [62]. The Notch pathway is also relevant as it constitutes an evolutionary conserved mechanism mediating cellcell communication between single-pass transmembrane receptors including, in our study, the targeted Jag1b and Notch1. Interestingly, roles for Notch signaling in fetal Leydig cells and in the communication between Sertoli cells and spermatogonia have been described, and in both mice and zebrafish, Notch signaling has been associated with initial or early stages of testis maturation $[12,63,64]$. Our findings therefore suggest that there might exist a previously unrecognized role for miRNAs in modulating the Notch pathway affecting early stages of testis maturation.

\section{Conclusion}

In this study we have identified miRNAs and their associated suppressed mRNAs/pathways, which could function either as activators or repressors of maturation. We identified both known and unknown miRNAs that potentially regulate pathways involved in the start of testis maturation.

Since a large proportion of the identified miRNAs has been associated with testis maturation in other vertebrates previously, conserved roles for these miRNAs seem possible $(m i R-101, m i R-137, m i R-18 a$, let7a/b/c, miR-92b, miR-20a, miR-25, miR-181a, miR-202, let7c/d/ $a$, miR-125b, miR-190a and miR-222a/b). On the other hand, we identified miRNAs not previously reported in 
the context of testis maturation (miR-new289, miR-15c, miR-20b, miR-30c, miR-724, miR-26b, miR-new271, miR217, miR-216a, miR-135a, miR-144, miR-145, miRnew194, miR-2184, miR-2188, miR-731, miR-8157 and the novel predicted miRNAs $n 2, n 7 a$ and $n 7 b$,). We have thus identified testicular miRNAs and their associated mRNAs, differentially expressed during the initiation of pubertal spermatogenesis. In comparison to previous papers reporting miRNAs associated to reproduction in fish, this paper utilizes an integrated approach considering differential expression of miRNAs and their predicted targeted mRNAs from the same tissue samples that were carefully selected for their different stages of development and activity, without showing important changes in the cellular composition of the tissue. However, the relatively low sample size may represent an uncertainty in this study. Further studies including functional reporter assays are therefore required to reach firm conclusions on target relations. Nevertheless, the presented data within this study provides a valuable resource and a starting point for future investigation of these matters.

\section{Methods}

\section{Fish and sampling}

The fish used in this study were Atlantic salmon postsmolts of defined genetic background (Aquagen strain). They were siblings or closely related, all from the same Aquagen strain. The fish were hatched and reared in freshwater indoor facilities at the Institute of Marine Research, the Matre Aquaculture Research Station, Matredal $\left(61^{\circ} \mathrm{N}\right)$, Norway, and transferred to sea cages after smoltification at 18 months of age, until January (6 months in sea water). Males used for the prepubertal and pubertal samples were all sampled in January while the immature fish were sampled in June. Fish were netted from the cage, immediately anesthetized with 6 ppt metomidate (Syndel, Victoria,B.C.) and weighed (total body weight). Blood (5 ml) was collected in heparinized syringes from the caudal vein. Subsequently, the fish were killed by cutting the medulla oblongata, after which gonad tissues were excised. Gonads were weighed for gonado-somatic index (GSI) determination (GSI = gonad weight $(\mathrm{g})$ "100/total body weight $(\mathrm{g})$ ) and central transversal pieces of testes tissue were cut with a scalpel blade. One piece of tissue was fixed in Bouins fixative for histological analysis, and another piece was immediately frozen in liquid nitrogen. Immunocytochemical detection of phosphorylated histone $\mathrm{H} 3$ was done according to established protocols [54]. Plasma samples were prepared according to Melo et al., 2015 [54] and quantification of 11-KT was performed by a radio-immuno assay [65]. In total 66 male fish were sampled, 3 were classified as immature, 3 as prepubertal and 3 as pubertal. A detailed description of samples used in the study can be found in Additional file 1.

\section{RNA extraction}

Total RNA including the small RNA fraction was extracted from $20 \mathrm{mg}$ of testis tissue samples, stored in liquid nitrogen, using the miRNeasy kit (Qiagen), according to the manufacturer's instructions. RNA samples were subsequently treated with TurboDNAse (Ambion) to remove any contaminating genomic DNA. RNA was analysed with a Agilent RNA 6000 Nano Kit (Agilent) and no traces of genomic DNA could be detected.

\section{mRNA and miRNA deep sequencing}

Preparation of cDNA for sequencing on the Illumina Hiseq2000 instrument was performed by the Norwegian Sequencing Centre using the Illumina TrueSeq small RNA library prep kit for miRNA and the TrueSeq RNA library prep kit for mRNA. mRNA and miRNA samples $(9$ samples each) were multiplexed and sequenced (100 bp read length) on 4 Illumina HiSeq2000 lanes. For mRNA paired end sequencing was utilized, whereas for miRNAs single end sequencing was used. Raw sequence files were demultiplexed by the Norwegian Sequencing Centre and provided to us as FASTQ files together with an initial FASTQC quality report [66]. The microRNA data and RNA-seq have been deposited to SRA, bioproject numbers PRJNA383545 and PRJNA380580, respectively.

\section{Fastq filtering and data analysis by miRDeep2}

miRNA reference files were downloaded from miRbase v21 [67] and processed by conversion to DNA sequences and removal of sequences containing sequence bases other than u,t,a,g,c. Fastq files trimmed by FastqMcf [68] were then analyzed using the miRdeep2 Perl scripts [69]. Filtered reads were mapped against a bowtie index of the Atlantic salmon genome (ICSASG_v2) using the miRdeep2 mapper.pl command. Next, in order to discover and quantify known and potential new miRNAs, the miRDeep2.pl command was executed, with miRNA reference files containing miRBase deposited miRNA sequences as well as previously identified novel salmon miRNA sequences $[32,33]$. The output of miRDeep2 were then cured and annotated by performing directional blast searches against miRBase v.21. Duplicate entries were removed, and repeat elements were removed by tandem repeats finder [70]. Novel miRNAs previously found by Bekaert et al. [33] are preceded by ssa-miRnew or ssa-miR-nov. Unannotated potentially novel miRNAs detected by the miRdeep2 pipeline were given a running number preceded by an $n$, indicating that these are potentially novel miRNAs (Additional file 3). 


\section{Gene expression analysis}

The gene expression table produced by miRdeep 2 was filtered to remove miRNA entries where the maximum depth of coverage among the libraries was less than 10 reads per sample and thereafter normalized by the Rlog function in the DESeq2 package [71]. The differentially expressed miRNAs reaching statistical significance, as determined by Benjamini \& Hochberg multiple test correction in DESeq2 ( $<<=0.05)$, were extracted and subjected to hierarchical and k-means clustering using the R-packages pheatmap and Mfuzz respectively [72, 73]. The expression levels of differentially expressed miRNAs can be found in Additional file 4. Paired end sequences from the mRNA seq were mapped with Bowtie2 [74] against the the Atlantic salmon genome (ICSASG_v2) and a raw count table was extracted with the use of SAMtools [75]. Entries where the maximum depth of coverage among the libraries was less than 10 reads per sample were discarded and differentially expressed genes (DEG) were computed with DESeq2, retrieving only DEG with a an absolute log2fold change $>=0.5$ and an adjusted $p$-value $<=0.05$. Mapping statistics and gene expression tables are provided as supplementary tables (Additional files 5 and 2).

\section{Prediction of miRNA targets and pathway analysis}

3 '-UTR's sequences for salmon mRNAs were extracted as the sequence spanning from CDS stop to the end of transcripts using a genbank flat file hosted at ncbi (GCF_000233375.1_ICSASG_v2_rna.gbff.gz). In cases where there were multiple annotations for a transcript, the longest 3'-UTR was retained. Sequences for each 3'UTR was then compiled to a multi fasta file. Based on this salmon 3 '-UTR reference multi fasta file, targets for differentially expressed miRNAs were predicted with miRMap [35]. Expression correlation between miRNAs and miRNA targets was calculated based on expression profiles from miRNAs as well as mRNAs from the same samples: An expression profile for averaged measurements across stages for each miRNA \& mRNA was made. Then using the python pandas.expanding_corr function, a pairwise Pearson correlation coefficient (1: perfectly correlated, -1 : anticorrelated) was calculated for these expression profiles. For downstream pathway analysis only mRNAs supported by a predicted miRNAmRNA relation (based on the 20\% top scoring miRmap target predictions for each miRNA) and with an anticorrelated expression (Pearson $<-0.5$ ), were used as input for KEGG analyses using the $\mathrm{R}$ ClusterProfiler package [76]. Target predictions and associated pathways were then visualized in Cytoscape [77]. In order to prevent excessive cluttering of the network, only a subset of these target relations consisting of the top 50\% scoring miRNA predictions for each pathway and with a negative expression correlation less than $-0,8$ were visualized. Nodes of the network (genes) were colored according to the strength of the negative correlation with their respective targeting miRNAs (Fig. 4).

\section{Quantitative PCR analysis}

We cross-validated the differential expression analysis with miRNA specific rt.-qPCRs using primers designed with miRprimer [78]. Conversion of RNA to cDNA was performed following the poly(A) polymerase (PAP) cDNA synthesis protocol, where non-polyadenylated RNA species like miRNAs are converted to a polyadenylated RNA intermediate by the use of E. coli poly(A) polymerase and primed with an anchored polydT primer containing an anchor site for a miRNA specific reverse primer in its $5^{\prime}$-end. The PAP cDNA synthesis and primer design was performed as described previously $[78,79]$. The cDNA was diluted 1 to 20 and $0.5 \mu \mathrm{l}$ of this diluted cDNA was utilized in a $6 \mu \mathrm{l}$ qPCR reaction with $3 \mu$ Fast SYBR green master mix (Applied Biosystems) and $3 \mu \mathrm{l}$ of a $0.5 \mu \mathrm{M}$ miRNA specific primer mix (Additional file 6). For about half of the miRNAs we could confirm an excellent correlation (Pearson correlation > $=0.98$ ) between the deep-sequencing and RTqPCR assays, and the majority of assays displayed a Pearson correlation greater than 0.8. For a few miRNAs, the RT-qPCR assays had Pearson correlation coefficients $<0.8$ (miR-135a-3p: 0.76, miR-135b-5p: 0.72, miR-181a4-3p: $0.56, m i R-202-5 p: 0.63, m i R-137-3 p: 0.64)$. It is likely that the primer design influences the success rate of a RT-qPCR assay and with miRNAs this has been reported to be particularly challenging due to the constrained positioning of the primers [78, 79]. Testicular transcript levels of igf3/amh were quantified as described previously [54].

\section{Additional files}

\section{Additional file 1: A detailed description of samples used in the study.} (DOCX 6 kb)

Additional file 2: Table S1. A complete overview of miRNAs obtained from cluster $I-V$,their high scoring predicted negatively correlated targets and KEGG enriched associated pathways. In a separate tab the 20\% highest scoring targets per miRNA (as assessed by miRmap score) not filtered by correlation is provided. Differential expressed mRNAs of pairwise comparisons between the immature, prepubertal and pubertal testis stages are also provided together with their respective Rlog normalized expression values in separate tabs. (XLSX 6839 kb)

Additional file 3: Table S2. miRNA reference file (sequences of mature and precursor miRNAs identified in this study). (XLSX 96 kb)

Additional file 4: Figure S1. Volcano plots of pairwise comparisons displaying differentially expressed miRNAs, their Log2fold changes and $p$-values. (PDF $242 \mathrm{~kb}$ )

Additional file 5: Table S3. Mapping statistics of mRNA and miRNA mapping. (XLSX 7 kb)

Additional file 6: Table S4. Primers. (PDF $39 \mathrm{~kb}$ ) 


\section{Abbreviations}

11-KT: 11-ketotestosterone; 3'-UTR: Three prime untranslated region; BPG: Brain-pituitary-gonad; CDS: Coding sequence; DHP: 17a,20ß-dihydroxy4-pregnen-3-one.; GSI: Gonado-somatic index; KEGG: Kyoto Encyclopedia of Genes and Genomes; miRNA: microRNA, RA: Retinoic acid

\section{Acknowledgements}

We would like to thank Anne Torsvik and Henk van de Kant for expert technical assistance.

\section{Funding}

We would like to thank the RCN project SALMOSTERILE (Grant number 221648) for funding this research.

\section{Availability of data and materials}

The raw microRNA and RNA-seq data have been deposited to SRA, bioproject numbers PRJNA383545 and PRJNA380580, respectively. Analyzed and filtered data are available in the published article and its supplementary information files.

\section{Authors' contributions}

Conceived and designed the experiments: KOS, AW, RBE, RWS, EA, GLT. Performed the experiments: KOS, DC, EA, LK, JB, RWS, GLT, TF. Analyzed the data: KOS, TF, RBE, RWS, AW. Contributed reagents/materials/analysis tools: EA, RWS, GLT, TF. Wrote the paper: KOS, RBE, RWS, AW. All coauthors approved the final manuscript.

\section{Ethics approval}

Approval of the experimental protocol of this experiment by the Norwegian Animal Research Authority (NARA) was not needed for this experimental approach. Consent to participate: Not applicable.

\section{Consent for publication}

Not applicable.

\section{Competing interests}

The authors declare that they have no competing interests.

\section{Publisher's Note}

Springer Nature remains neutral with regard to jurisdictional claims in published maps and institutional affiliations.

\section{Author details}

${ }^{1}$ Institute of Marine Research, Postboks 1870 Nordnes, 5817 Bergen, Norway. ${ }^{2}$ Reproductive Biology group, Department of Biology, Faculty of Science, Utrecht University, Padualaan 8, 3584 CH Utrecht, The Netherlands.

\section{Received: 17 July 2017 Accepted: 9 October 2017}

Published online: 18 October 2017

\section{References}

1. Schulz RW, de França LR, Lareyre J-J, Le Gac F, LeGac F, Chiarini-Garcia H, et al. Spermatogenesis in fish. Gen Comp Endocrinol. 2010;165:390-411.

2. Zohar Y, Muñoz-Cueto JA, Elizur A, Kah O. Neuroendocrinology of reproduction in teleost fish. Gen Comp Endocrinol. 2010;165:438-55.

3. Levavi-Sivan B, Bogerd J, Mañanós EL, Gómez A, Lareyre JJ. Perspectives on fish gonadotropins and their receptors. Gen Comp Endocrinol. 2010;165: 412-37

4. Taranger GL, Carrillo M, Schulz RW, Fontaine P, Zanuy S, Felip A, et al. Control of puberty in farmed fish. Gen Comp Endocrinol. 2010;165:483-515.

5. Miura T, Miura C, Konda Y, Yamauchi K. Spermatogenesis-preventing substance in Japanese eel. Dev Camb Engl. 2002;129:2689-97.

6. Miura T, Ohta T, Miura Cl, Yamauchi K. Complementary deoxyribonucleic acid cloning of spermatogonial stem cell renewal factor. Endocrinology. 2003;144:5504-10.

7. Sawatari E, Shikina S, Takeuchi T, Yoshizaki GA. Novel transforming growth factor-Beta superfamily member expressed in gonadal somatic cells enhances primordial germ cell and spermatogonial proliferation in rainbow trout (Oncorhynchus Mykiss). Dev Biol. 2007;301:266-75.
8. Assis LHC, Crespo D, Morais RDVS, França LR, Bogerd J, Schulz RW. INSL3 stimulates spermatogonial differentiation in testis of adult zebrafish (Danio rerio). Cell Tissue Res. 2015;363:579-88.

9. Nóbrega RH, RDV de S M, Crespo D, de Waal PP, de França LR, Schulz RW, et al. Fsh stimulates Spermatogonial proliferation and differentiation in zebrafish via lgf3. Endocrinology. 2015:156:3804-17.

10. Skaar KS, Nóbrega RH, Magaraki A, Olsen LC, Schulz RW, Male R. Proteolytically activated, recombinant anti-mullerian hormone inhibits androgen secretion, proliferation, and differentiation of spermatogonia in adult zebrafish testis organ cultures. Endocrinology. 2011;152:3527-40.

11. Nicol B, Guiguen Y. Expression profiling of Wnt signaling genes during gonadal differentiation and gametogenesis in rainbow trout. Sex. Dev. Genet. Mol. Biol. Evol. Endocrinol. Embryol. Pathol. Sex Determ. Differ. 2011; 5:318-29.

12. Crespo D, Assis LHC, Furmanek T, Bogerd J, Schulz RW. Expression profiling identifies Sertoli and Leydig cell genes as Fsh targets in adult zebrafish testis. Mol Cell Endocrinol. 2016:437:237-51.

13. Martinović-Weigelt D, Wang R-L, Villeneuve DL, Bencic DC, Lazorchak J, Ankley GT. Gene expression profiling of the androgen receptor antagonists flutamide and vinclozolin in zebrafish (Danio Rerio) gonads. Aquat Toxicol Amst Neth. 2011;101:447-58.

14. Blázquez M, Medina P, Crespo B, Gómez A, Zanuy S. Identification of conserved genes triggering puberty in European sea bass males (Dicentrarchus Labrax) by microarray expression profiling. BMC Genomics. 2017; 18:441.

15. Kim VN, Han J, Siomi MC. Biogenesis of small RNAs in animals. Nat Rev Mol Cell Biol. 2009;10:126-39.

16. Bizuayehu TT, Babiak I. MicroRNA in teleost fish. Genome Biol Evol. 2014;6: 1911-37.

17. Hilz S, Modzelewski AJ, Cohen PE, Grimson A. The roles of microRNAs and siRNAs in mammalian spermatogenesis. Dev. Camb. Engl. 2016;143:3061-73.

18. Bartel DP. MicroRNAs: target recognition and regulatory functions. Cell. 2009;136:215-33.

19. Buchold GM, Coarfa C, Kim J, Milosavljevic A, Gunaratne PH, Matzuk MM. Analysis of MicroRNA Expression in the Prepubertal Testis. PLoS ONE 2010 [cited 2015 Mar 12];5. Available from: http://www.ncbi.nlm.nih.gov/pmc/ articles/PMC3012074/

20. Papaioannou MD, Nef S. microRNAs in the testis: building up male fertility. J Androl. 2010;31:26-33.

21. Romero Y, Meikar O, Papaioannou MD, Conne B, Grey C, Weier M, et al. Dicer1 depletion in male germ cells leads to infertility due to cumulative meiotic and spermiogenic defects. PLoS One. 2011;6:e25241.

22. Wu Q, Song R, Ortogero N, Zheng H, Evanoff R, Small CL, et al. The RNase III enzyme DROSHA is essential for microRNA production and spermatogenesis. J Biol Chem. 2012;287:25173-90.

23. Tong M-H, Mitchell DA, McGowan SD, Evanoff R, Griswold MD. Two miRNA clusters, Mir-17-92 (Mirc1) and Mir-106b-25 (Mirc3), are involved in the regulation of spermatogonial differentiation in mice. Biol Reprod. 2012;86: 72.

24. Rakoczy J, Fernandez-Valverde SL, Glazov EA, Wainwright EN, Sato T, Takada S, et al. MicroRNAs-140-5p/140-3p modulate Leydig cell numbers in the developing mouse testis. Biol Reprod. 2013;88:143.

25. Hung P-S, Liu C-J, Chou C-S, Kao S-Y, Yang C-C, Chang K-W, et al, miR-146a enhances the oncogenicity of oral carcinoma by concomitant targeting of the IRAK1, TRAF6 and NUMB genes. PLoS One. 2013:8:e79926.

26. Huszar JM, Payne CJ. MicroRNA 146 (Mir146) modulates Spermatogonial differentiation by retinoic acid in mice. Biol Reprod. 2013:88:15.

27. Kasimanickam VR, Kasimanickam RK, Dernell WS. Dysregulated microRNA Clusters in Response to Retinoic Acid and CYP26B1 Inhibitor Induced Testicular Function in Dogs. PLoS ONE 2014 [cited 2015 Mar 12];9. Available from: http://www.ncbi.nlm.nih.gov/pmc/articles/PMC4049822/

28. Jing J, Wu J, Liu W, Xiong S, Ma W, Zhang J, et al. Sex-biased miRNAs in gonad and their potential roles for testis development in yellow catfish. PLoS One. 2014:9:e107946.

29. Bizuayehu TT, Fernandes JMO, Johansen SD, Babiak I. Characterization of novel precursor miRNAs using next generation sequencing and prediction of miRNA targets in Atlantic halibut. PLoS One. 2013;8:e61378.

30. Bizuayehu T, Babiak J, Norberg B, Fernandes JMO, Johansen SD, Babiak I. Sex-biased miRNA expression in Atlantic halibut (Hippoglossus Hippoglossus) brain and gonads. Sex Dev Genet Mol Biol Evol Endocrinol Embryol Pathol Sex Determ Differ. 2012;6:257-66. 
31. Johansen I, Andreassen R. Validation of miRNA genes suitable as reference genes in $\mathrm{PPCR}$ analyses of miRNA gene expression in Atlantic salmon (Salmo Salar). BMC Res Notes. 2014;8:945.

32. Andreassen R, Worren MM, Høyheim B. Discovery and characterization of miRNA genes in Atlantic salmon (Salmo Salar) by use of a deep sequencing approach. BMC Genomics. 2013;14:482.

33. Bekaert M, Lowe NR, Bishop SC, Bron JE, Taggart JB, Houston RD. Sequencing and characterisation of an extensive Atlantic Salmon (Salmo Salar L.) MicroRNA repertoire. PLoS One. 2013;8:e70136.

34. Farlora R, Valenzuela-Miranda D, Alarcón-Matus P, Gallardo-Escárate C. Identification of microRNAs associated with sexual maturity in rainbow trout brain and testis through small RNA deep sequencing. Mol Reprod Dev. 2015;82(9):651-62.

35. Vejnar CE, Zdobnov EM. MiRmap: comprehensive prediction of microRNA target repression strength. Nucleic Acids Res. 2012;40:11673-83.

36. Norfo R, Zini R, Pennucci V, Bianchi E, Salati S, Guglielmelli P, et al. miRNA-mRNA integrative analysis in primary myelofibrosis CD34+ cells: role of miR-155/JARID2 axis in abnormal megakaryopoiesis. Blood. 2014;124:e21-32.

37. Summerer I, Hess J, Pitea A, Unger $K$, Hieber L, Selmansberger M, et al. Integrative analysis of the microRNA-mRNA response to radiochemotherapy in primary head and neck squamous cell carcinoma cells. BMC Genomics. 2015;16:654.

38. Wotschofsky Z, Gummlich L, Liep J, Stephan C, Kilic E, Jung K, et al. Integrated microRNA and mRNA signature associated with the transition from the locally confined to the metastasized clear cell renal cell carcinoma exemplified by miR-146-5p. PLoS One. 2016;11:e0148746.

39. Jonas S, Izaurralde E. Towards a molecular understanding of microRNAmediated gene silencing. Nat Rev Genet. 2015;16:421-33.

40. Kraft C, Herzog F, Gieffers C, Mechtler K, Hagting A, Pines J, et al. Mitotic regulation of the human anaphase-promoting complex by phosphorylation. EMBO J. 2003:22:6598-609.

41. Holt JE, Pye V, Boon E, Stewart JL, García-Higuera I, Moreno S, et al. The APC/C activator FZR1 is essential for meiotic prophase I in mice. Dev. Camb. Engl. 2014;141:1354-65.

42. Baker DJ, Jeganathan KB, Cameron JD, Thompson M, Juneja S, Kopecka A, et al. BubR1 insufficiency causes early onset of aging-associated phenotypes and infertility in mice. Nat Genet. 2004:36:744-9.

43. Nakanishi M, Ando H, Watanabe N, Kitamura K, Ito K, Okayama H, et al. Identification and characterization of human Wee1B, a new member of the Wee1 family of Cdk-inhibitory kinases. Genes cells devoted Mol. Cell. Mech. 2000:5:839-47.

44. DeBruhl H, Wen H, Lipsick JS. The complex containing drosophila Myb and $\mathrm{RB} / \mathrm{E} 2 \mathrm{~F} 2$ regulates cytokinesis in a histone $\mathrm{H} 2 \mathrm{Av}$-dependent manner. Mol Cell Biol. 2013;33:1809-18.

45. Presslauer C, Tilahun Bizuayehu T, Kopp M, Fernandes JMO, Babiak I. Dynamics of miRNA transcriptome during gonadal development of zebrafish. Sci. Rep 2017;7. Available from: http://www.ncbi.nlm.nih.gov/pmc/ articles/PMC5338332/

46. Sangiao-Alvarellos S, Manfredi-Lozano M, Ruiz-Pino F, León S, Morales C, Cordido F, et al. Testicular expression of the Lin28/let-7 system: hormonal regulation and changes during postnatal maturation and after manipulations of puberty. Sci Rep. 2015;5:15683.

47. Lau K, Lai KP, Bao JYJ, Zhang N, Tse A, Tong A, et al. Identification and Expression Profiling of MicroRNAs in the Brain, Liver and Gonads of Marine Medaka (Oryzias melastigma) and in Response to Hypoxia. PLoS ONE 2014 [cited 2015 Mar 12];9. Available from: http://www.ncbi.nlm.nih.gov/pmc/ articles/PMC4211694/

48. Chen J, Cai T, Zheng C, Lin X, Wang G, Liao S, et al. MicroRNA-202 maintains spermatogonial stem cells by inhibiting cell cycle regulators and RNA binding proteins. Nucleic Acids Res. 2017;45:4142-57.

49. Josso N, Racine C, di Clemente N, Rey R, Xavier F. The role of anti-Müllerian hormone in gonadal development. Mol Cell Endocrinol. 1998;145:3-7.

50. Pfennig F, Standke A, Gutzeit HO. The role of Amh signaling in teleost fishmultiple functions not restricted to the gonads. Gen Comp Endocrinol. 2015;223:87-107.

51. Gilchrist GC, Tscherner A, Nalpathamkalam T, Merico D, LaMarre J. MicroRNA expression during bovine oocyte maturation and fertilization. Int J Mol Sci. 2016;17:396.

52. Pitetti J-L, Calvel P, Zimmermann C, Conne B, Papaioannou MD, Aubry F, et al. An essential role for insulin and IGF1 receptors in regulating sertoli cell proliferation, testis size, and FSH action in mice. Mol. Endocrinol. Baltim. MD. 2013:27:814-27.

53. Wang W, Liu W, Liu Q, Li B, An L, Hao R, et al. Coordinated microRNA and messenger RNA expression profiles for understanding sexual dimorphism of gonads and the potential roles of microRNA in the steroidogenesis pathway in Nile tilapia (Oreochromis Niloticus). Theriogenology. 2016;85:970-8.

54. Melo MC, van Dijk P, Andersson E, Nilsen TO, Fjelldal PG, Male R, et al. Androgens directly stimulate spermatogonial differentiation in juvenile Atlantic salmon (Salmo salar). Gen Comp Endocrinol. 2015;211:52-61.

55. Miura T, Higuchi M, Ozaki Y, Ohta T, Miura C. Progestin is an essential factor for the initiation of the meiosis in spermatogenetic cells of the eel. Proc Natl Acad Sci U S A. 2006;103:7333-8.

56. Liu G, Luo F, Song Q, Wu L, Qiu Y, Shi H, et al. Blocking of progestin action disrupts spermatogenesis in Nile tilapia (Oreochromis Niloticus). J Mol Endocrinol. 2014:53:57-70.

57. Chen SX, Bogerd J, Schoonen NE, Martijn J, de Waal PP, Schulz RWA Progestin (17a,20ß-dihydroxy-4-pregnen-3-one) stimulates early stages of spermatogenesis in zebrafish. Gen Comp Endocrinol. 2013; 185:1-9.

58. Mäkelä J-A, Saario V, Bourguiba-Hachemi S, Nurmio M, Jahnukainen K, Parvinen $\mathrm{M}$, et al. Hedgehog signalling promotes germ cell survival in the rat testis. Reprod. Camb. Engl. 2011;142:711-21.

59. Coultas L, Bouillet $\mathrm{P}$, Loveland KL, Meachem S, Perlman H, Adams JM, et al. Concomitant loss of proapoptotic $\mathrm{BH} 3$-only $\mathrm{BCl}-2$ antagonists Bik and Bim arrests spermatogenesis. EMBO J. 2005;24:3963-73.

60. Jesus TT, Oliveira PF, Sousa M, Cheng CY, Alves MG. Mammalian target of rapamycin (mTOR): a central regulator of male fertility? Crit Rev Biochem Mol Biol. 2017:52:235-53.

61. Li Z, Lu J, Sun X, Pang Q, Zhao Y. Molecular cloning, mRNA expression, and localization of the G-protein subunit Galphaq in sheep testis and epididymis. Asian-Australas J Anim Sci. 2016;29:1702-9.

62. Shen G, Wu R, Liu B, Dong W, Tu Z, Yang J, et al. Upstream and downstream mechanisms for the promoting effects of IGF-1 on differentiation of spermatogonia to primary spermatocytes. Life Sci. 2014; 101:49-55.

63. Garcia TX, Farmaha JK, Kow S, Hofmann M-C. RBPJ in mouse Sertoli cells is required for proper regulation of the testis stem cell niche. Dev. Camb. Engl. 2014;141:4468-78.

64. Defalco T, Saraswathula A, Briot A, Iruela-Arispe ML, Capel B. Testosterone levels influence mouse fetal Leydig cell progenitors through notch signaling. Biol Reprod. 2013;88:91.

65. Mayer I, Borg B, Schulz R. Conversion of 11-ketoandrostenedione to 11ketotestosterone by blood cells of six fish species. Gen Comp Endocrinol. 1990;77:70-4.

66. Andrews S. FastQC A Quality Control tool for High Throughput Sequence Data. Available from: http://www.bioinformatics.babraham.ac.uk/projects/ fastqc/

67. Griffiths-Jones S, Grocock RJ, van Dongen S, Bateman A, Enright AJ. miRBase: microRNA sequences, targets and gene nomenclature. Nucleic Acids Res. 2006;34:D140-4.

68. Services EG. FastgMcf. 2017. Available from: https://github.com/ ExpressionAnalysis/ea-utils

69. Friedländer MR, Mackowiak SD, Li N, Chen W, Rajewsky N. miRDeep2 accurately identifies known and hundreds of novel microRNA genes in seven animal clades. Nucleic Acids Res. 2012:40:37-52

70. Benson G. Tandem repeats finder: a program to analyze DNA sequences. Nucleic Acids Res. 1999;27:573-80.

71. Love MI, Huber W, Anders S. Moderated estimation of fold change and dispersion for RNA-seq data with DESeq2. Genome Biol. 2014:15:550.

72. Kolde R. pheatmap: Pretty Heatmaps [Internet]. 2015. Available from: http:// CRAN.R-project.org/package=pheatmap

73. Futschik M. Mfuzz: soft clustering of time series gene expression data [internet]. 2015. Available from: http://www.sysbiolab.eu/software/R/Mfuzz/ index.html

74. Langmead B, Salzberg SL. Fast gapped-read alignment with bowtie 2. Nat Methods. 2012;9:357-9.

75. Li H, Handsaker B, Wysoker A, Fennell T, Ruan J, Homer N, et al. The sequence alignment/map format and SAMtools. Bioinforma. Oxf. Engl. 2009; 25:2078-9.

76. Yu G, Wang L-G, Han Y, He Q-Y. ClusterProfiler: an R package for comparing biological themes among gene clusters. OMICS J. Integr Biol. 2012;16:284-7. 
77. Shannon P, Markiel A, Ozier O, Baliga NS, Wang JT, Ramage D, et al. Cytoscape: a software environment for integrated models of biomolecular interaction networks. Genome Res. 2003;13:2498-504.

78. Busk PKA. Tool for design of primers for microRNA-specific quantitative RTqPCR. BMC Bioinformatics. 2014;15:29.

79. Balcells I, Cirera S, Busk PK. Specific and sensitive quantitative RT-PCR of miRNAs with DNA primers. BMC Biotechnol. 2011;11:70.

Submit your next manuscript to BioMed Central and we will help you at every step:

- We accept pre-submission inquiries

- Our selector tool helps you to find the most relevant journal

- We provide round the clock customer support

- Convenient online submission

- Thorough peer review

- Inclusion in PubMed and all major indexing services

- Maximum visibility for your research

Submit your manuscript at www.biomedcentral.com/submit
Biomed Central 